\title{
Kuvunga: Timbre, interlocking, and composite melodies in Zambian Luvale ngoma
}

\author{
Jason Reid Winikoff ${ }^{1 \dagger}$ \\ ${ }^{1}$ School of Music, The University of British Columbia, Vancouver, British Columbia, Canada \\ ${ }^{\dagger}$ Corresponding author: jason.winikoff@alumnni.ubc.ca \\ Published 16 December 2021; https://doi.org/10.18061/FDMC.2021.0052 \\ Author video presentation and/or other conference material: https://doi.org/10.17605/OSF.IO/F9J6S
}

\begin{abstract}
This paper is an attempt to explicate the concept of "kuvunga," an important term to Zambian Luvale drummers. Drums kuvunga when they produce a composite melody. While these composites have long been understood as vital to African percussion, the processes that create them - both performed and perceived - have been relatively ignored. To help illustrate these processes, I introduce new analytic terminology that organizes rhythms along timbral lines. When these types of rhythms interact in specific ways, the drums kuvunga. In this study I will explain how Luvale musicians achieve this interlocking resultant by manipulating timbre through technical control. I combine research on auditory stream segregation, African musicology, and ethnographic methods to show that making drums kuvunga involves performed and perceived attention to timbre. Ultimately, my analysis draws attention to the vitality of timbre in African percussion, explicates the emergence of an important perceptual construct, and provides a new set of analytic tools.
\end{abstract}

KEYWORDS: timbre, Luvale, perception, percussion, Zambia

\section{Introduction}

In scholarly discourse and general belief, drumming has often been reduced to a solely rhythmic artform. This is perhaps most manifest in sub-Saharan African music where, as Agawu (1995) convincingly argues, scholars' preoccupation with rhythm has led to an excessive othering of African musicians - rhythmic complexity separates Africans from everyone else. With this "invention of African rhythm" comes an oversight of other sonic parameters in drumming such as pitch and timbre. Although scholars like Nzewi (1974) have begun to draw attention to the melodic dimensions of African drumming, timbre has remained comparatively absent from this discussion. This is unfortunate as composite drum melodies are vital to these music systems, and the performed and perceived processes that create them heavily involve timbre. What follows in this study is an analysis of drum timbre and its impact on these composites in Zambian Luvale percussion.

At the heart of this study is an examination of the Luvale verb "kuvunga." Luvale musicians often invoke the word kuvunga when discussing supporting drums. On the surface, this verb refers to making drums sound good together. A deeper probing of the word, however, reveals information about emic timbral aesthetics. Ultimately, I present an understanding of this term as composite melodic-rhythms constructed through performed and perceived attention to timbre.

Kubik (1994) labeled a Kigandan xylophone version of this phenomenon the IP Effect. Fiagbedzi (1980) demonstrated how compositional practices yield the effect in Anlo-Ewe dance-drumming. In this study I argue that a Luvale version of the IP Effect, encoded in the concept of kuvunga, emerges not solely because of how the music was composed, but also in conjunction with performance of the composition and, importantly, perceptual processes of reorganization.

The Luvale people predominately live in present-day northwestern Zambia, eastern Angola, and southern Democratic Republic of the Congo. They are closely related to the Chokwe, Mbunda, and Luchazi peoples and together make up a relatively homogenous regional cultural grouping. Although relatives of the Luvale have been the focus of much musicological literature, research on Luvale music is relatively scarce. The little that does exist primarily focuses on the voice with only one study dedicated to Luvale drums (Tsukada, 1997).

Percussion ensembles play important roles in Luvale traditional festivals, initiation ceremonies, healing rituals, and entertainment. The Luvale percussion ensemble consists of a lead drum (ngoma pwita, ngoma ya shina, or jingoma jya kachacha), the mikakaji (two sticks struck against the body of a drum), and two to three supporting drums: ngoma yahakachi, ngoma yakusongo, and occasionally ngoma yakasumbi. [1] Supporting drums essentially produce three different sounds: an open tone and two different closed tones (a muffled stroke and a slap stroke). Proper hand technique is vital to producing these distinct timbres, and proper timbre is essential for making drums kuvunga.

\section{Method}

This study is based on ethnographic methods involving participant-observation, interviews, and experience as 
both a student and performer of this music. I conducted on-site research in Zambia in 2013-2014, 2015, 2017, and 2019. I also investigate the concept of kuvunga through deep readings of dictionary definitions, music analysis including the introduction of new analytical terminology, and research in the fields of African (ethno)musicology and cognitive psychology.

When investigating timbre, Fales (2002) stresses the importance of distinguishing between the acoustic and perceptual domains. I follow her lead by splitting my timbral inquiry into two levels: that which deals with the production of acoustic stimuli and that of perception.

The terminology in my theory classifies drum parts along timbral and melodic lines. These categorical terms provide information on technique, the instrument itself, and how the part is likely to interact with other musical ideas. This study utilizes three of these categories: durarhythm, timbre-rhythm, and melo-rhythm.

A dura-rhythm (short for durational-rhythm) is a part whose events are only differentiated (if at all) through rhythmic means; there are no timbral or melodic dissimilarities. This usually implies that each moment of attack involves the same technique and part of the drum. In the Luvale repertoire, dura-rhythms are almost exclusively used by supporting drums. Figure 1 (A-B) provides two examples of common Luvale durarhythms. Note that each example only includes one timbre (note-head) and one pitch (vertical position).

A timbre-rhythm is a musical idea in which a drummer rhythmically moves between notes that vary only in timbre (and, perhaps, duration); it is a timbral unfolding of notes, not a melodic one. These different timbres are produced through differences in technique and/or the instrument itself (e.g., hitting a different part of the drum or using a different kind of stick). Timbrerhythms usually have a hierarchy of tones, what Knight (1974) calls "a carefully balanced system of strong and weak accents...[a] system of inherent accents" (p. 29). In this way, the other timbre(s) support or embellish the dominant one. Frequently the open tones provide the instrument's main musical contribution to the ensemble's gestalt. Other timbres thus help provide musical interest or, as Locke (1998) notes, help the performer. Timbre-rhythms are quite common in both Luvale supporting and lead drumming. In examples CD of Figure 1, note the variety of note-heads despite the sometimes-absent durational variety. [2]

A melo-rhythm (short for melodic-rhythm) is a musical idea in which a drummer rhythmically moves between various pitches of the same timbre. [3] As melo-rhythms only include one timbre, they usually involve only one type of hand technique. Since a single ngoma drum is essentially only capable of producing one definite pitch (that of the open stroke), melorhythms usually require several drums. In the Luvale repertoire they are thus uncommon for supporting instruments, instead only featured in a specialized genre of lead drumming/dance known as Kachacha. In Figure 1 (E-F) note that while rhythm and pitch may change, each event has the same note-head and, thus, timbre.

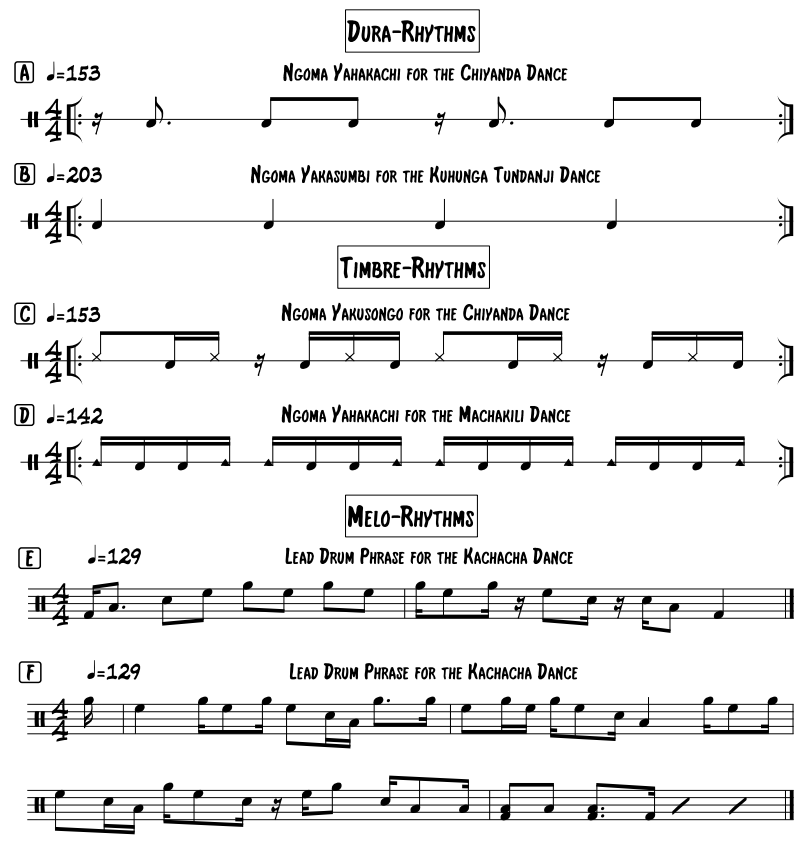

Figure 1: Examples of my terminology in the Luvale percussion ensemble repertoire. (E-F) show lead drum phrases Kapalu Lizambo often plays. Normal noteheads represent open strokes. " $X$ " note-heads represent slaps. Triangular note-heads represent muffled tones.

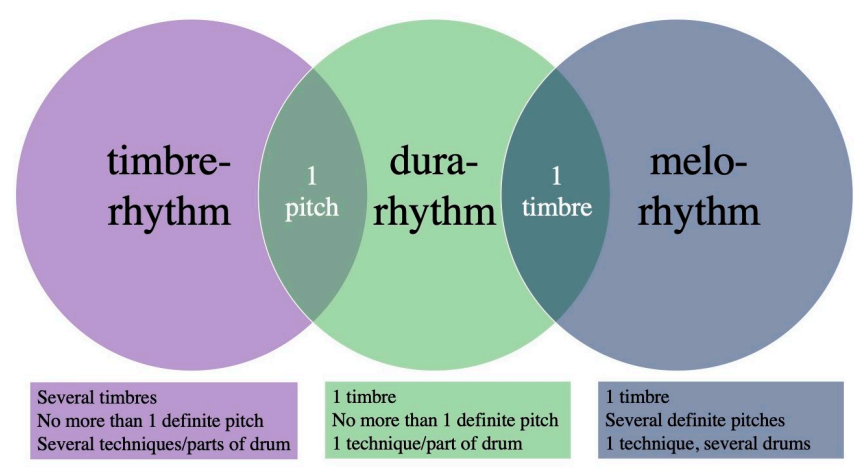

Figure 2: A visual aid for understanding my analytic terminology. The three categories of parts are organized along timbral and melodic lines. 


\section{Results}

In one of the only Luvale dictionaries, Horton (1975) defines kuvunga: "Carry the air of a song. Play the first or treble drum...To do something turn and turn about, alternate with one another or others" (p. 434). Here kuvunga involves playing a single drum in a way that carries the music whilst alternating with another.

In an interview, Kapalu Lizambo (arguably the most respected performer in this cultural region) partially contradicted Horton, instead stating that kuvunga involves the relationship between several drums/drummers. To Lizambo, this relationship is so vital that no one else (e.g., lead drummer, singer, dancer) can enter until the drums kuvunga. He explained, "You hear the kuvunga for three, two $n g o m a . .$. if the kuvunga is going in the same way or good like that." I interjected, "So if the drums are marrying." He eagerly agreed, happy to hear me using a metaphor he (and others) taught me years before. "Yeah. Now, ok, that's kuvunga, it's now straight. Then you can now come in. Because you can't come in if the kuvunga is not straight." (personal communication, August 1, 2019). Lizambo also mentioned that the different timbres (open and closed) of several drums connect as one in the process of kuvunga. "Kuvunga...that's different sounds. Yes. So the kuvunga is coming to the different $n g o m a . .$. so that one now you are connected for that sound" (personal communication, August 1, 2019). But how do several drums provide different timbres in a way that unifies the foundational layer?

In another interview, William Vunda, the leader of the Chota Culture Group, spoke on the importance of alternating timbres in kuvunga. He explained: "Opening and closing, you are the one who is giving your friend time also to breathe with their drums. So once you just open it - continuously open it - then these guys, the other drummers won't close...they won't kuvunga well. Yes. It's you who is supposed to open and close, open and close. So once you open, then them they close. You you close, then them they open. So it's like changing" (personal communication, July 17, 2019). Perhaps the alternation that Horton refers to is one of timbre.

If we place the words of Horton, Lizambo, and Vunda together, we can make several observations about the act of kuvunga. (1) It is the essence of a piece in that it both carries the music and is the foundation for other layers (e.g., lead drumming, song, dance). (2) Several drums are involved. (3) It involves not just the presence of several timbres, but the structured alternation of them. (4) The alternation of different tone colors leads to a unified whole. This process of unification, though, is not just created in the productive domain through technical control but also in the perceptual domain through specific cognitive processes.

Although the principles of kuvunga that I outline here apply to many pieces of Luvale music, I will focus on one example. Mokolo is an important and popular item of Luvale dance music. Example A of Figure 3 represents the supporting drum parts for that dance while Example B illustrates listeners' aural image of those same drums. In the remaining sections I will show how performance of Example A leads to perception of Example B. I argue that this entire chain of events, from drum stroke to perception, is the process of kuvunga.

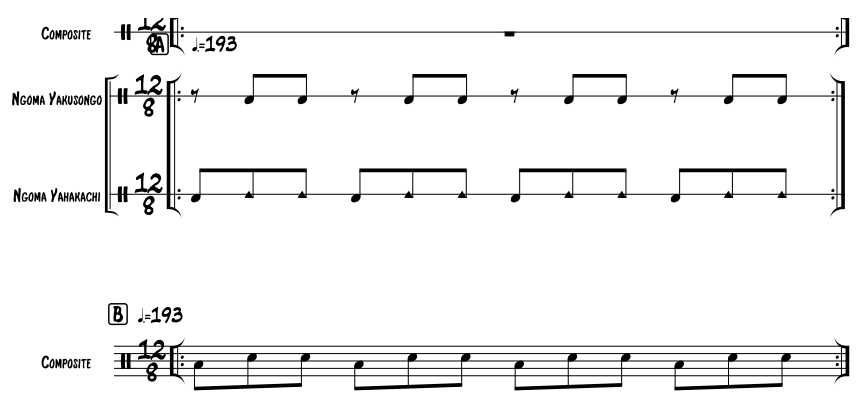

Figure 3: (A) The supporting drums for Mokolo and (B) their perceived composite.

\section{Discussion}

In the Mokolo example, the supporting drums do not rhythmically interlock as they frequently overlap; each ngoma yakusongo event occurs at the same time as a ngoma yahakachi note. Despite this, these drums still kuvunga well. Structured timbral alternation allows the listener to only perceive open strokes. This happens because open tones mask simultaneously occurring closed tones. Fales and McAdams (1994) define "masking" as the phenomenon when one timbre is perceived more stronger than other simultaneously occurring timbres. Bregman (1990) notes that lower tones (like an open stroke) are more likely to mask higher ones (e.g., slap or muffled stroke). He adds that masking is strengthened when the sources are physically close. This is the case with supporting drums as they are always adjacent in the ensemble's setup. An upward spread of masking, aided by the ensemble's physical orientation, allows for simultaneously sounding drums to contribute to the music in a cooperative manner.

An interlock, then, can work even if there is rhythmic overlap. The key lies in vertical timbral differentiation. At a moment of simultaneous onset, one drummer 
"breathes" through an open tone while the other gives space through a closed (slapped or muffled) timbre. This works not only because the closed stroke has a different timbre from the open one, but also because it is a secondary timbre - one that cedes its power to the primary, open strokes. This is a defining feature of a timbre-rhythm. The ngoma yahakachi of Mokolo plays a timbre-rhythm and its muffled tones are secondary to open strokes. This allows perception of every simultaneously occurring open tone of ngoma yakusongo's dura-rhythm. The principles of masking allow these dura- and timbre-rhythms to cooperate and are partially responsible for the perception of the composite melo-rhythm represented in Figure 3 (B).

Lizambo's point on how supporting drums must be unified in order for any other layer to enter is supported by cognitive psychology research. Bregman (1990) found that background notes (in our case, those provided by supporting drums) must be precise in order for the foreground to have its effect.

Although melo-rhythms are relatively rare in the productive domain of Luvale traditional music, they are ubiquitous in the perceptual domain and vital to the concept of kuvunga. The other two types of drum parts (dura- and timbre-rhythms) interact with each other to create a composite melo-rhythm. These composites result from perceptual grouping along timbral lines. But what must happen in the productive domain for the listener to reorganize the material as such in the perceptual domain? Scholars agree that notes from different sources are likely to segregate from their own musical units and form a new stream if the target notes: have a similar timbre (Bregman, 1990; Bregman \& Pinker, 1978; Kubik, 1994, 2010; Goodchild \& McAdams, 2018), are temporally close (Bregman 1990), are close in frequency (Bregman 1990; Kubik 2010; Goodchild \& McAdams, 2018), are part of a fast sequence (Bregman, 1990), emerge from sources that are physically near each other (Bregman, 1990; Goodchild \& McAdams, 2018), or belong to repeating sequences (Bregman, 1990; Kubik, 2010). Luvale supporting drums meet these criteria as different drums are capable of producing sounds with similar quality, are temporally close due to tight interlocking, are tuned closely and relative to each other, are played quickly, are set up next to each other, and repeat their parts.

Musical listening (as opposed to non-musical listening) is especially conducive to this type of auditory stream segregation along timbral lines. Fales (2002) notes that, when listening to music, we ignore source characteristics and, subconsciously, naturally hear the whole. Locke (1998) argues that acoustically prominent "bounce [or open] strokes constitute the player's "speech," his contribution to the ensemble's musical conversation...the contribution to the call-andresponse" (pp. 41-42, 47). Since tonal events segregate from noise-like events (Bregman \& Pinker, 1978), the pitched open strokes of each Luvale supporting drum will segregate from the noisy slaps, instead joining the other drums' open strokes of similar timbre. In order for these strokes to contribute as they do, they must segregate from and mask other types of strokes. When all of these findings are placed in conversation with one another, we can draw some valuable conclusions in a chain that lead us to a melo-rhythmic composite.

(1) When listening to music, we naturally perceive some version of the sum, not the individual parts. (2) Since the process of musical perception is distanced from that of source recognition, listeners will subconsciously seek out auditory stream segregation based on acoustic similarities; the sources of these sonic events are secondary at best. (3) If these sonic events are fast, repeated, close in timbre, pitch, time, and physical proximity then we are likely to group them together. (4) The sonic events we are likely to integrate into one unit are the open strokes (which segregate from and mask the other timbres). This means our composite is a series of pitches that have the same timbre, likely produced by similar techniques. (5) The composite is a melo-rhythm, born when several drummers contribute at least one pitch each with a similar timbre.

The composite melo-rhythm that is so vital to African drumming is a result of musicians' execution of timbre and listeners' timbrally-sensitive perceptual processes. In the Mokolo example, timbre- and durarhythms contribute to the perceptual melo-rhythm. The entirety of dura-rhythms contributes while only the primary, open tones of timbre-rhythms are included. The listener's mind extracts the open tones - creating a drummed version of Kubik's inherent pattern or Nzewi's complementary melo-rhythmic theme. This perceived melo-rhythm is the unified whole, born out of the alternation of several drums' timbres, that is the essence of the piece. Providing the appropriate acoustic stimuli to yield a composite melo-rhythm foundation in the perceptual domain is the process of kuvunga.

\section{Conclusion}

In this study I attempted to present an understanding of the Luvale musical term kuvunga. I looked to the productive domain to see how technique influences and reinforces Luvale drummers' definitions of the word. 
This revealed that kuvunga cannot just refer to interlocking rhythms because of these moments of simultaneous attack, because of these notions of open and closed timbres. If we ignore timbre, the parts do not interlock; there would be frequent overlap and no space for the drums to speak and breathe. Drummers must also alternate timbres. Dura- and timbre-rhythms are differentiated and defined through timbral means. By carefully relating these types of parts to one another in the productive domain, Luvale drummers help suggest a composite melo-rhythm for listeners in the perceptual domain. This composite, I argue, is the essence of kuvunga, and supporting drums that kuvunga are the foundation of Luvale music. Kuvunga references a specific type of auditory stream segregation, a drummed inherent timbre-pattern that is articulated through timbral control. An analysis of the processes, both performed and perceived, that yield composite melorhythms reveals the vitality of timbre to drumming.

This study also offers just a glimpse of what we can learn through ethnography and by placing our work within the frame of non-Western epistemologies. Cognitive psychology would benefit from investigating timbre beyond both the lab and Western culture. I suggest that we incorporate ethnographic methods and data, if nothing else, as a collaborative counterpart to quantitative-data driven studies. I call on scholars to expand the cultural and geographic scopes of their work and to apply their findings to non-Western case-studies.

\section{Acknowledgements}

This study would not have been possible without the aid of Kapalu Lizambo, William Vunda, the members of Lenga Navo and Chota culture groups, the Likumbi lya Mize Cultural Association, and the late Senior Chief Ndungu VIII. I would also like to thank Michael Tenzer for his supervision and help with early drafts. A version of this paper was presented at the 2020 SEM meeting.

\section{End Notes}

1. These drum names are not fully standardized and vary among cultural groups and areas of Luvale settlement. Ensembles may also contain additional instruments such as a njenje, ngezo, and/or sangu/jisangu.

2. Notes with different timbres may also have a slight perceived difference in pitch (this perceived difference may be a result of timbre). Because of this, I not only represent timbral difference through note-heads, but also through vertical position.

3. My "melo-rhythm" is inspired by Nzewi's (1974) concept of "melo-rhythmic essence." Although similar, our terms should not be used interchangeably. His term is applied to (what I term) "timbre-rhythms" that also have perceived melodic form. Nzewi's term serves his purposes as it is not part of a larger theory. My theory, however, is concerned with the different effects that result from timbral and melodic variation. As such, my term is more specific and restricted in its use.

\section{References}

Agawu, K. (1995). The Invention of "African Rhythm". Journal of the American Musicological Society, 48(3), 380-395. https://doi.org/10.2307/3519832

Bregman, A. S. (1990). Auditory Scene Analysis: The Perceptual Organization of Sound. MIT Press. https://doi.org/10.7551/mitpress/1486.001.0001

Bregman, A. S., \& Pinker, S. (1978). Auditory Streaming and the Building of Timbre. Canadian Journal of Psychology, 32(1), 19-31. https://doi.org/10.1037/h0081664

Fales, C. (2002). The Paradox of Timbre. Ethnomusicology, 46(1), 56-95. https://doi.org/10.2307/852808

Fales, C., \& McAdams, S. (1994). The Fusion and Layering of Noise and Tone: Implications for Timbre in African Instruments. Leonardo Music Journal, 4, 69-77. https://doi.org/10.2307/1513183

Fiagbedzi, N. (1980). A Preliminary Inquiry into Inherent [sic] Rhythms in Anlo Dance Drumming. Journal of the Performing Arts, 1(1), 82-92.

Goodchild, M., \& McAdams, S. (2018). Perceptual Processes in Orchestration. In E. Dolan \& A. Rehding (Eds.), The Oxford Handbook of Timbre. Oxford University Press. https://doi.org/10.1093/oxfordhb/ 9780190637224.013.10

Horton, A. E. (1975). A Dictionary of Luvale. Rahn Bros. Print \& Lithographing Co.

Knight, R. (1974). Mandinka Drumming. African Arts, 7(4), 24-35. https://doi.org/10.2307/3334885

Kubik, G. (1994). Theory of African Music (Vol. 1). The University of Chicago Press.

Kubik, G. (2010). Theory of African Music (Vol. 2). The University of Chicago Press. https://doi.org/10.7208/ chicago/9780226456928.001.0001

Locke, D. (1998). Drum Gahu: An Introduction to African Rhythm. White Cliffs Media.

Nzewi, M. (1974). Melo-Rhythmic Essence and Hot Rhythm in Nigerian Folk Music. The Black Perspective in Music, 2(1), 23-28. https://doi.org/10.2307/1214145

Tsukada, K. (1997). Drumming, Onomatopoeia and Sound Symbolism Among the Luvale in Zambia. In J. Kawada (Ed.), Cultures Sonores d'Afrique (pp. 349-393). Institute for the Study of Languages \& Cultures of Asia \& Africa, Tokyo University of Foreign Studies. 